\title{
Envejecimiento y factores asociados a la calidad de vida de los adultos mayores en el Estado de México*
}

\author{
Zuriel Soria-Romero y Bernardino Jaciel Montoya-Arce
}

\author{
Universidad Autónoma del Estado México, México
}

\section{Resumen}

En este artículo se aborda la relación que existe entre las condiciones sociodemográficas, económicas y de salud con la calidad de vida de los adultos mayores que participaron en la Encuesta sobre Envejecimiento Demográfico en el Estado de México. Se usó la técnica de Análisis de Componentes Principales para calcular el índice de calidad de vida y considerar variables objetivas y subjetivas agrupadas en cuatro dimensiones: bienestar, ingreso, condiciones de la vivienda y condiciones de salud. Del análisis se desprendió que más de la mitad de la población en estudio carece de condiciones adecuadas en su calidad de vida y se muestran los factores relacionados, tales como escolaridad, estado civil y condición de derechohabiencia.

Palabras clave: Calidad de vida, envejecimiento, análisis de componentes principales; Estado de México.

\section{Abstract}

Aging and factors associated with quality of life for elderly people in State of Mexico

This article presents an analysis of the relationship between socio-demographic, economic and health conditions with the quality of life of elderly who participated in the Survey on Demographic Aging in the State of Mexico. Using the technique of Principal Component Analysis, an index of quality of life considering objective and subjective variables grouped into four areas: well-being, income, housing conditions and health conditions was calculated. From the analysis it emerged that more than half of the study population lacks adequate conditions that affect their quality of life and shows that the factors that greatly related to the quality of life of elderly are the level of schooling, marital status and rightfulness condition.

Key words: Quality of life, aging, principal component analysis, State of Mexico.

\footnotetext{
* Este artículo forma parte de los productos derivados del proyecto de investigación titulado "Diagnóstico Integral de la Situación Actual del Envejecimiento Demográfico en el Estado de México", que tuvo como instrumento de análisis la Encuesta sobre Envejecimiento Demográfico en el Estado de México (ESEDEM 2008). Esta investigación se realizó en el Centro de Investigación y Estudios Avanzados de la Población de la Universidad Autónoma del Estado de México. Los autores agradecen al doctor Alfonso Mejía Modesto y al maestro Hugo Montes de Oca Vargas, la atención que tuvieron en leer este texto y hacer las recomendaciones que consideraron pertinentes para mejorar su contenido.
} 


\section{INTRODUCCIÓN}

$\mathbf{L}$

a transición demográfica se entiende como el cambio de tasas altas a tasas bajas de crecimiento natural (Ramírez, 2002). Esto, a

su vez, implica un cambio en la estructura poblacional que se ve reflejado en un continuo y acelerado proceso de envejecimiento poblacional. El incremento en la esperanza de vida, la reducción de la mortalidad y los avances en materia de salud impactan en una mayor longevidad que exige tomar una serie de medidas a fin de lograr un envejecimiento sano y de calidad.

El objetivo de este texto es estudiar la calidad de vida y el envejecimiento, con la finalidad de conocer, mediante un índice, qué factores se relacionan en mayor medida con el bienestar de los adultos mayores del Estado de México. El artículo se organiza en cuatro apartados: i) envejecimiento de la población, en el que se define el envejecimiento poblacional como objeto de estudio y se describe cómo viven los adultos mayores en el país y en la entidad mexiquense; ii) calidad de vida; se mencionan algunos enfoques de estudio que ha tenido el tema; ii) metodología: se explica el modelo estadístico aplicado para construir un índice de bienestar de los adultos mayores; y iv) resultados: se detallan las condiciones de vida de la población envejecida, así como su relación con variables específicas de tipo sociodemográficas, económicas y de la salud.

\section{ENVEJECIMIENTO DE LA POBLACIÓN}

El envejecimiento es entendido como

un proceso que se vive desde el nacimiento y se caracteriza por diferentes cambios en niveles físicos, mentales, individuales y colectivos; es además un proceso natural, irreversible y no necesariamente ligado a estereotipos y debe verse como una etapa del ciclo vital llena de potencial para crecer y continuar aprendiendo (Organización Panamericana de la Salud, 2002: 2).

Existen ciertas diferencias respecto a la edad que demarca el envejecimiento, puesto que hay diversas perspectivas: cronológica, biológica, psíquica o social. La Organización de las Naciones Unidas (ONU) establece la edad de 60 años para considerar que una persona es adulta mayor, aunque en los países desarrollados se considera que empieza a los 65 años. Sin embargo, para efectos de esta investigación y bajo la perspectiva cronológica, y dado que el envejecimiento va ligado a cambios en la posición de la persona en la sociedad, se considera como adulto mayor al individuo 
de 60 años o más, asociándolo también al empleo y la jubilación, además, en apego a la Legislación del Estado de México que también entiende por adultos mayores a hombres y mujeres a partir de esta edad (Ley del Adulto Mayor del Estado de México, 2008).

El envejecimiento demográfico es resultado de un proceso de transición caracterizado por un incremento tanto en números porcentuales como absolutos del grupo poblacional de 60 años o más, producto de la disminución en las tasas de fecundidad y mortalidad, y con ello el incremento en la esperanza de vida gracias a los adelantos tecnológicos en medicina y al mayor acceso a los servicios de salud que han implementado los gobiernos desde hace décadas. El envejecimiento ocurre en todas las regiones del mundo, pero aumenta con mayor rapidez en países con diferentes niveles de desarrollo, incluidos aquellos que también tienen una proporción importante de población joven. A continuación se presentan algunos datos importantes referentes a este tema por parte de la Organización Mundial de la Salud (OMS, 2012), a fin de mostrar por qué es considerado un tema importante en el mundo:

- Las proyecciones de población indican que en el año 2050 habrá en el mundo cerca de 400 millones de personas con 80 años o más. Igualmente, habrá por primera vez más personas de edad avanzada que niños menores de 15 años.

- Actualmente Japón es el único país del mundo cuya población en edades avanzadas representa más de 30 por ciento de su población total. Hacia 2050 habrá 64 países que compartan esta situación.

- El número de adultos mayores en África aumentará de 54 a 213 millones.

- En la actualidad, las principales causas de muerte en esta población son las enfermedades cardiacas, respiratorias y los accidentes cerebrovasculares, mientras que las principales causas de discapacidad son el déficit visual, demencia, pérdida auditiva y artrosis. Éstas irán en aumento.

- La OMS considera que todos los profesionales sanitarios deberían recibir formación sobre las cuestiones relacionadas con el envejecimiento.

Resulta importante mencionar algunos datos de la población en México con el fin de contextualizar el fenómeno del envejecimiento demográfico y, de acuerdo con los datos de la Encuesta Intercensal, 2015, en la Tabla 1 y en la Tabla 2, respectivamente, se observa que en 2015 más de la décima parte de la población en el país corresponde a personas de 60 años o más, 
mientras que en el Estado de México nueve de cada 100 pertenecen a este grupo etario. De acuerdo con las proyecciones del Consejo Nacional de Población (CONAPO) se espera que dichas cifras vayan en aumento; además de que la población envejecida femenina es mayor que la masculina, por lo que habrá que analizar cómo enfrentan las mujeres este proceso.

Tabla 1: Población adulta por sexo en México, 2015

\begin{tabular}{lrrrr}
\hline & Hombres & Mujeres & Total & $\%$ \\
\hline Población total & 58013739 & 61430245 & 119443984 & - \\
Población de 60 años o más & 5750299 & 6686022 & 12436321 & 10.41 \\
\hline Fuente: elaboración propia con base en resultados de la Encuesta Intercensal, 2015. &
\end{tabular}

Tabla 2: Población adulta por sexo en el Estado de México, 2015

\begin{tabular}{lrrrr}
\hline & Hombres & Mujeres & Total & $\%$ \\
\hline Población total & 7826440 & 8345140 & 16171580 & - \\
Población de 60 años o más & 693384 & 824041 & 1517425 & 9.38 \\
\hline
\end{tabular}

Fuente: elaboración propia con base en resultados de la Encuesta Intercensal, 2015.

A pesar de que en 2015 el Estado de México fue una de las entidades con menor porcentaje de personas de 60 años o más, ocupa el lugar 25 de todas las entidades de la República Mexicana con 9.38 por ciento, como se observa en la Figura 1; sin embargo, en la Figura 2, se aprecia que es la entidad con mayor número absoluto de este rango de personas, alberga más de la doceava parte de ellas en el país y en los próximos años se presentará un incremento sostenido en la proporción de adultos mayores en referencia con la población total de la entidad, por lo que se prevé una situación futura con gran cantidad de gente en edades avanzadas, lo que implica enfrentar retos para los gobiernos, instituciones y principalmente la familia y sociedad, tales como el desarrollo de infraestructura y equipamientos necesarios para atender las demandas de este grupo, también se enfrentarán a cuestiones como pensiones, derechohabiencia, salud, dependencia, entre otros. México es uno de los países que atraviesa una fase de plena y acelerada transición demográfica y esta situación es considerada por demógrafos, como Ordorica (2012), como el tema demográfico del siglo XXI.

En décadas anteriores el reto para la ciencia era sumar años en la esperanza de vida de las personas; esto se está logrando gracias a los avances en medicina, programas preventivos y mayor acceso a los servicios de salud. 
Envejecimiento y factores asociados a la calidad de vida de los adultos ... /Z. SORIA y B.J. MONTOYA

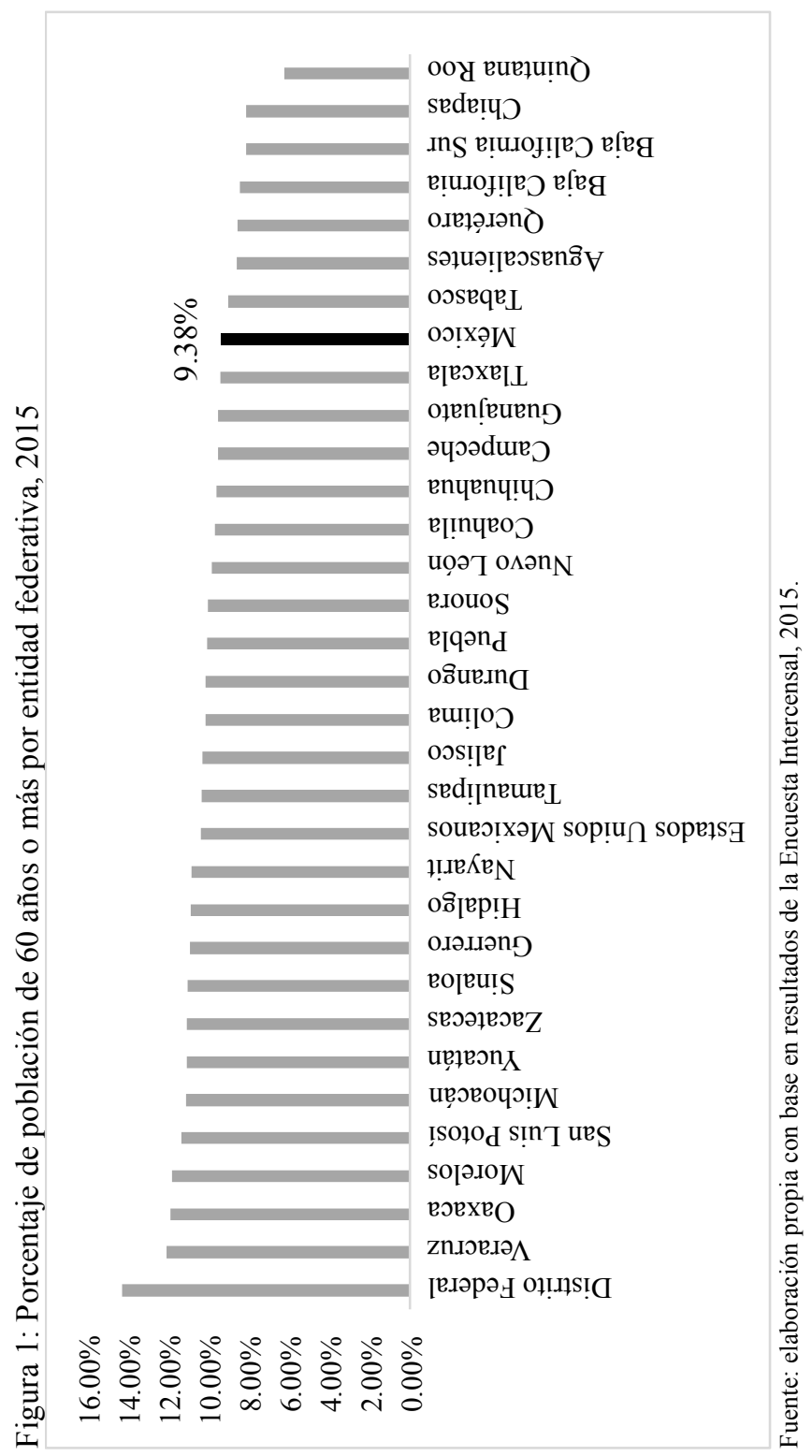




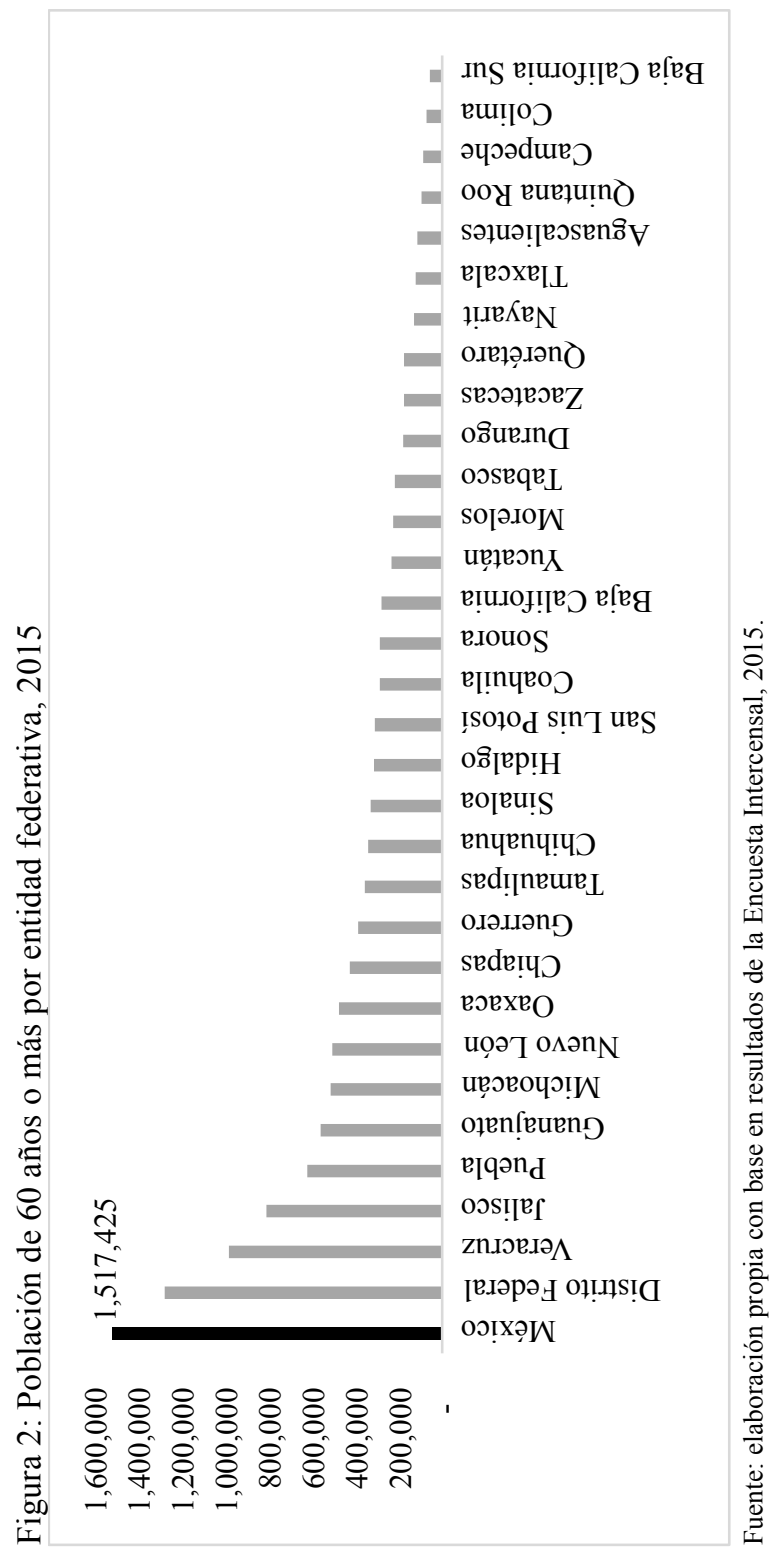


En México, de acuerdo con el Instituto Nacional de Estadística y Geografía (INEGI), en 1930 la esperanza de vida para las mujeres era de 35 años y para los hombres de 33. Para 2010 este indicador fue de 77 para ellas y 71 para ellos; en 2014 se ubicó en poco más de 77 años, casi igual para las mujeres y en 72 para los hombres. Sin embargo, el reto actual no sólo debe consistir en aumentarle años a la vida, sino en mejorarla e incrementar el bienestar de la población. Referente a la esperanza de vida, Welti (2013) afirma que éste no ha ido a la par de modificaciones en las instituciones que conlleven a que el adulto mayor viva en condiciones aceptables.

De acuerdo con la Comisión Económica para América Latina y el Caribe (CEPAL, 2002), México vive una fase de envejecimiento demográfico moderadamente avanzado; esto se observa en las cifras de la Encuesta Intercensal que indican que en 2015 había un total de 119443984 habitantes en el país, de los cuales, 10.4 por ciento corresponden a adultos mayores de 60 años o más. En 2015 se registraron 38 adultos con este rango de edad por cada 100 menores de 15 , y por cada 100 en edad económicamente activa había 16 de 60 años o más.

Uno de los indicadores más empleados para estudiar el crecimiento de este grupo es el índice de envejecimiento que relaciona la cantidad de personas adultas mayores con la cantidad de niños y jóvenes, y permite observar la velocidad con la que crece cada conjunto; otro es el índice de dependencia, que relaciona a la población inactiva o económicamente dependiente con la activa. La tendencia de éstos permite elaborar proyecciones $\mathrm{y}$, aunque es importante recordar que el futuro es incierto puesto que son muchos los factores que lo determinan:

Las proyecciones de la población actualmente representan un instrumento fundamental de la política de población, ya que permiten anticiparse a las demandas sociales y evaluar las diferentes trayectorias que surgirían de afectarse o mantenerse las tendencias actuales de las variables que inciden en el monto, estructura y dinámica demográfica (Ordorica, 2010: 33).

Entonces, de acuerdo con el Consejo Nacional de Población (Conapo, 2016) se espera que para 2030, en el país, la población de 60 años o más alcance 14.8 por ciento del total; el índice de envejecimiento crecerá pues se pronostica que habrá 63 personas en este rango por cada 100 menores de 15 años. Asimismo, en cuanto al índice de dependencia se esperan 24 adultos mayores por cada 100 en edad económicamente activa.

De acuerdo con Tuirán (1999) muchas de las instituciones económicas y sociales han estado fundadas en el supuesto de que el número de ni- 
ños y adolescentes es significativamente mayor que el de adultos mayores. Los cambios en la distribución por edades que se prevén en los próximos años incidirán en la formación de nuevos comportamientos, demandas y necesidades. La población envejecida exigirá en mayor medida atención médica y psicológica de calidad; en el ámbito económico, la población activa deberá mantener a un número creciente de adultos mayores dependientes, garantizándoles un ingreso básico y con ello una vida digna. En los servicios, tal vez se requieran menos guarderías y servicios pediátricos y más geriatras, gerontólogos, oncólogos, cardiólogos y especialistas en la atención de enfermedades crónico-degenerativas. Es necesario, desde ahora, iniciar programas de educación y capacitación a modo de que todos los profesionales sanitarios reciban formación para atender las necesidades de este grupo de personas; además se debe crear la infraestructura para la atención adecuada a sus demandas.

Es importante considerar el tema del envejecimiento dentro de los planes de desarrollo puesto que

la situación actual y las proyecciones permiten inferir que en el siglo XXI, México compartirá, al igual que la mayor parte del mundo, un proceso de envejecimiento que deberá ser tomado muy en cuenta y desde ahora si se quiere aspirar a un futuro con planeación y perspectivas adecuadas en beneficio de este grupo poblacional y de la sociedad en general (Ham, 1998: 32).

\section{Envejecimiento en el Estado de México}

En el Estado de México, al igual que en el resto del país, la transición demográfica encamina a una modificación en la estructura por edades y a un proceso gradual de envejecimiento. De acuerdo con los resultados de la Encuesta Intercensal 2015, el estado registró una población total de poco más de 16 millones de habitantes, cifra similar a la suma de la población de 11 estados del país, que lo convierte en el territorio con el mayor número de habitantes; por ello, la importancia de estudiar el fenómeno del envejecimiento en esta entidad.

En 2030, según las proyecciones del Conapo (2016), el Estado de México seguirá siendo el más poblado, con poco más de 20 millones de habitantes, mientras que la Ciudad de México tendrá casi 8.5 millones, de modo que en el centro del país seguirá prevaleciendo una fuerte concentración de población y entre ambas entidades se concentrará la quinta parte de la población nacional. 
El grupo de adultos mayores en la entidad mexiquense pasó de 7.5 por ciento en 2010 a 9.3 por ciento en 2015 , mientras que la población de cero a 14 años registró un descenso, al pasar de 28.9 a 26.5 por ciento en el mismo periodo. El Conapo (2016) ha estimado un aumento gradual y para 2030 prevé que la población con 60 años o más será de 14.7 por ciento ( Figura 3). Por ello es conveniente desde ahora tomar las medidas necesarias para hacer frente a las implicaciones económicas, sociales, políticas, territoriales y culturales que se tendrán en años futuros, además de garantizar que la población en edad avanzada disfrute de una vejez digna.

Figura 3: Proyección del porcentaje de adultos mayores en el Estado de México, 2017-2030

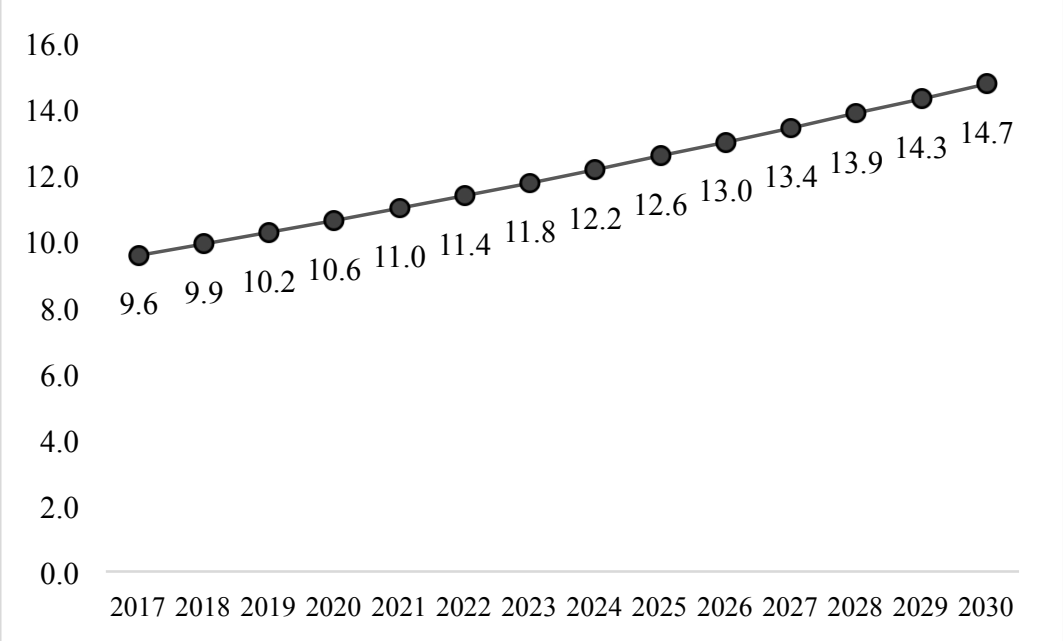

Fuente: elaboración propia con base en Proyecciones del Conapo 2017-2030.

\section{Calidad de vida}

El concepto de calidad de vida es reciente, de acuerdo con Cuenca (2008) esta expresión fue usada por primera vez por el presidente de Estados Unidos, Lyndon Johnson en 1964 en un discurso acerca de los planes de salud. Anteriormente, el concepto ya se había empleado bajo un enfoque economicista y cuantitativo, pero ahora tiene una gran especialización en diferentes campos como el médico, económico, cultural, religioso, ecológico, entre otros; sin embargo, de acuerdo con García (2008) no hay una medición cuantitativa única, porque depende de la conceptualización que se tenga de la misma, de los indicadores contemplados y de la información estadística disponible. En este sentido, por calidad de vida se entiende: 
una expresión ligada a múltiples factores, tanto objetivos como subjetivos, con el bienestar y la satisfacción por la vida y cuya evidencia esta intrínsecamente relacionada con su propia experiencia, su salud y su grado de interacción social y ambiental (Vera, 2007: 285).

La calidad de vida se relaciona con diferentes propósitos como la evaluación de las necesidades de las personas y sus niveles de satisfacción, la evaluación de resultados de programas y servicios, la dirección y guía en la provisión de estos servicios y en la formulación de políticas nacionales e internacionales dirigidas a la población en general y a otras más específicas (Robles et al., 2010).

Este tema ha sido estudiado desde diferentes enfoques tanto objetivos como subjetivos, Schwartzmann, mencionado por Vinnacia y Orozco (2005), asocia la calidad de vida a diversos dominios o dimensiones, lo define como un juicio subjetivo de satisfacción o de felicidad alcanzado o, en términos simples, como un sentimiento de bienestar personal; sin embargo, añade la importancia de relacionar lo subjetivo con indicadores objetivos biológicos, psicológicos, comportamentales y sociales.

\section{Calidad de vida en la vejez}

De acuerdo con Zetina (1999), por lo general, a la vejez se le percibe como una edad de deterioro asociada a la disminución en las capacidades físicas y mentales, a diferencia de otras etapas de la vida que se consideran de crecimiento y desarrollo. En contraparte, la Organización Panamericana de la Salud (2002) la concibe como un proceso natural, de crecimiento y aprendizaje.

En décadas anteriores la percepción que se tenía de los adultos mayores era como fuente de sabiduría, conocimiento y fortaleza, incluso, era importante contar con uno de ellos en la familia; empero, ahora la percepción ha cambiado y es asociada principalmente con dependencia o enfermedad, de modo que las proles cada vez menos se quieren hacer cargo de ellos.

De acuerdo con la Encuesta Nacional sobre Discriminación en México (Enadis, 2010), los adultos mayores son considerados el cuarto grupo de población vulnerable a la discriminación, ante los pueblos indígenas, personas de color y homosexuales quienes ocupan los primeros sitios. La discriminación, según Romero (2005), está caracterizada por los atributos negativos de la improductividad, la ineficiencia, la enfermedad y la decadencia general, así entonces, el envejecimiento lejos de verse como una etapa natural dentro del ciclo de vida, se liga a un proceso lleno de amenaza y degradación, por lo cual dicha condición no es esperada con felicidad. 
Este grupo de personas experimenta cambios físicos, psicológicos y sociales, que los convierte en una población vulnerable, entendiendo por vulnerabilidad:

El proceso multidimensional y multicausal, en el que confluyen simultáneamente la exposición a riesgos, la incapacidad de respuesta y adaptación de individuos, hogares o comunidades, los cuales pueden ser heridos, lesionados o dañados ante cambios o permanencia de situaciones externas y/o internas que afectan su nivel de bienestar y el ejercicio de sus derechos (Busso, 2005: 16).

Los adultos mayores presentan desventajas como la mayor exposición a enfermedades, sobre todo crónico-degenerativas e incapacitantes, muestran también condiciones de dependencia económica y pobreza, pérdida de las capacidades físicas y mentales y disminución en su grado de autonomía y adaptabilidad. En relación con lo anterior y de acuerdo con Ordorica (2010), se estima que entre 2010 y 2050 el número de personas en edades avanzadas que presentará impedimentos físicos pasará de 3 a 15 millones, de modo que se multiplicará por cinco en este periodo, esto sin considerar el posible incremento en la esperanza de vida, lo que acentuará dicha cifra.

La calidad de vida también resulta de la unión de diferentes factores como la vivienda, los bienes, el ingreso, el vestido, la alimentación, la educación, el apoyo social percibido e incluso variables sociodemográficas como la edad y el sexo. Vera (2007) concluye en su investigación que tanto para esta gente como para su familia la calidad de vida también se asocia a elementos secundarios como los cuidados y una protección digna, además del respeto hacia su condición de persona y de ser humano, pues genera ambientes de dependencia que repercuten en el hogar.

Así, para este estudio y de acuerdo con la definición del Grupo para la Valoración de la Calidad de Vida de la Organización Mundial de la Salud, "la calidad de vida se considera como la percepción del individuo sobre su posición en la vida dentro del contexto cultural y del sistema de valores en el que vive y con respecto a sus metas, expectativas, normas y preocupaciones" (OMS, 2002: 98). Basados en ellos para medir la calidad de vida del adulto mayor en esta investigación se consideran elementos tanto objetivos como subjetivos y se agrupan en cuatro áreas: i) condiciones de la vivienda, ii) ingreso, iii) bienestar subjetivo y iv) condiciones de Salud.

\section{Condiciones de la vivienda}

La vivienda es un "espacio delimitado generalmente por paredes y techos de cualquier material, con entrada independiente, que se construyó para 
la habitación de personas, o que al momento del levantamiento censal se utiliza para vivir" (INEGI, 2005: 120).

Ésta es, entonces, un lugar físico donde la gente desarrolla actividades cotidianas y se protege de las inclemencias del tiempo. Mientras mejor sea, mayor será el impacto positivo en el bienestar de las personas, puesto que aquéllas construidas con materiales resistentes como tabique, ladrillo, block o cemento, brindan una mayor protección que las que están hechas con láminas de cartón, asbesto o metálicas, madera o adobe e incluso materiales de desecho (INEGI, 2005).

La vivienda se asocia con la calidad de vida de quienes la habitan, ya que es uno de los satisfactores básicos para la supervivencia, además se le concibe como un espacio que debe proveer a sus ocupantes protección, higiene, privacidad, comodidad y la seguridad de encontrarse en una situación de propiedad que proporcione la certeza de disponer de ella en el presente y futuro (INEGI, 2005).

\section{Ingreso}

Es un tema que adquiere suma importancia como aspecto primordial en la calidad de vida en la vejez, pues durante esta etapa disminuyen las capacidades físicas y mentales que limitan el desarrollo de actividades remuneradas y generalmente se deja de percibir un ingreso (Nava y Ham, 2014). Estudios como los de Guzmán y Huenchuan (2006) demuestran que la familia juega un papel importante en el sostén económico de los adultos mayores y la que proporciona los factores necesarios para su atención y desarrollo integral; no obstante, el respeto y el apoyo hacia estas personas se pierde cada vez más.

La seguridad económica se entiende como la capacidad de disponer y usar de forma independiente una determinada cantidad de recursos económicos, suficiente para asegurar una buena calidad de vida (Guzmán, 2003).

De acuerdo con la Encuesta Nacional de Empleo y Seguridad Social (ENESS, 2013) sólo una cuarta parte de los adultos mayores está pensionada, lo que equivale a 26.1 por ciento, mientras que la población estudiada es de 18 por ciento del total que tiene acceso a esta prestación, situación que va ligada a los bajos niveles educativos que presenta la mayoría; el sexo masculino es el más beneficiado con 75 por ciento.

La situación referente al bajo acceso a un sistema de pensiones y a la inviabilidad financiera en el largo plazo ha producido en los últimos años reformas en materia de seguridad social y laboral que, de acuerdo con Welti (2013), incidirán de manera negativa en las condiciones de vida 
de este grupo de personas residentes en México, porque carecen de recursos suficientes para acceder a la satisfacción de sus necesidades y que, en materia de salud, requieren de montos considerables de recursos para ser atendidos.

\section{Bienestar subjetivo}

Si bien es importante la inclusión de elementos subjetivos en la medición de la calidad de vida, un punto importante a considerar es si éstos realmente reflejan la calidad de vida de las sociedades. En este sentido, se ha señalado que las percepciones están medidas por aspectos psicológicos, de tal manera que el hecho de tener buenas condiciones objetivas de bienestar no siempre coincide con altas condiciones subjetivas, y a la inversa.

Lo anterior se debe a las expectativas que implica el hecho de que las personas se adapten a sus circunstancias de vida y, por tanto, que se ajusten a esas condiciones (Sen, 1985, mencionada por Ochoa, 2011). El concepto de calidad de vida

ha ido evolucionando en los últimos años desde una concepción básicamente materialista, en la que se distinguían los aspectos objetivos de nivel de vida, pasando hacia una perspectiva donde los aspectos subjetivos constituyen el elemento fundamental (Puig et al., 2011: 13).

La importancia de considerar estas variables en el estudio, de acuerdo con Ovalle y Martínez (2006), se debe a que los individuos enfocan su calidad de vida en los dominios que los rodean, más que en los valores materiales que poseen; en particular, autores como Ochoa (2011) han demostrado que los indicadores objetivos más utilizados, como el nivel de ingreso per cápita, en efecto están relacionados con este aspecto, pero no en su totalidad, debido a que existe un amplio espacio para la discusión acerca de los elementos que la conforman, propone incorporar en el estudio, además de los dominios económicos, dimensiones subjetivas que arrojen información sobre qué tan satisfechos están con la vida.

Igualmente, Millán sostiene que:

La calidad de vida se relaciona con la percepción de felicidad. ¿Es usted feliz? Si la respuesta es $s i ́$ entonces se puede admitir que su calidad de vida es buena, ya que lo contrario - la infelicidad - generaría desazón, malestar o insatisfacción. La felicidad, entendida como la reflexión afectiva que se hace sobre el grado de satisfacción con la vida, y que a veces se expresa como la consecución de aquello que se anhela, contribuirá sin duda, a tener una buena calidad de vida (Millán, 2011: 8). 
En general, existe un acuerdo en señalar que la felicidad se relaciona con la salud y con la duración de la vida. Por otra parte, Eamon O'Shea, mencionado por Vera (2007), sostiene que la calidad de vida del adulto mayor es satisfactoria, bienestar subjetivo y psicológico, desarrollo personal y diversas representaciones de lo que constituye una buena vida, y que se debe indagar, preguntándole cómo da sentido a su propia vida.

\section{Condiciones de salud}

Los adelantos tecnológicos y en medicina para prolongar la vida deben ir acompañados por una mejor calidad de esa vida. En una población envejecida aparecerán con mayor prevalencia las denominadas patologías edaddependientes, es decir, enfermedades cuya incidencia se incrementa con la edad, como es el caso de las crónico-degenerativas y por ello la importancia de garantizar la existencia y acceso a servicios de atención médica adecuados que atiendan sus demandas.

Además, la discapacidad afectará la facultad del adulto mayor para realizar las actividades de la vida cotidiana: en primer lugar las consideradas como complejas o avanzadas, como el ocio y de relaciones sociales; en segundo, las instrumentales, como utilizar el teléfono o manejar el dinero y, por último, las básicas, es decir, la capacidad para vestirse, asearse o alimentarse.

La dependencia es el factor más importante a la hora de que el sujeto valore su calidad de vida, ya que de alguna manera genera en él la necesidad de un apoyo por parte de un tercero, que en muchos casos no está disponible y que siempre es limitador de su propia autonomía (Millán, 2011: 1).

\section{Metodología}

En este trabajo se aplicó la técnica estadística de Análisis de Componentes Principales (ACP), cuyo objetivo es reducir la información sobre un conjunto de individuos, de los cuales se han tomado diversas observaciones sobre varias de sus características. Esta técnica permitió construir índices de calidad de vida para las personas de 60 años o más que participaron en la Encuesta sobre Envejecimiento Demográfico en el Estado de México en 2008 (ESEDEM 2008), realizada por el Centro de Investigación y Estudios Avanzados de la Población (CIEAP). Es importante mencionar que dicha encuesta tiene la desventaja del tiempo que ha transcurrido desde que se levantó; sin embargo, no existe información estadística disponible más reciente que permita hacer una medición de calidad de vida de los adultos 
mayores de la entidad mexiquense. Una vez que se obtuvieron dichos índices se elaboraron cuadros de contingencia considerando variables sociodemográficas, económicas y de la salud con el fin de conocer la situación y la relación que presentan con el nivel de calidad de vida calculado para los adultos mayores.

\section{Descripción de la base de datos}

El Centro de Investigación y Estudios Avanzados de la Población (CIEAP) se propuso llevar a cabo un detallado diagnóstico del envejecimiento demográfico en el Estado de México y como instrumento de análisis empleó la ESEDEM, 2008.

El método de análisis aplicado en la investigación fue el cuantitativo descriptivo, a través de un diseño de marco muestral que se conformó teniendo como base todas las áreas geoestadísticas básicas y localidades rurales del Estado de México. La muestra se seleccionó a través de un esquema probabilístico polietápico, con probabilidad proporcional al número de viviendas particulares, tanto en áreas urbanas como localidades rurales (menos de 2500 habitantes), y consistió en la aplicación de cuestionarios de hogar e individual en campo. En cada uno de los hogares seleccionados, el número de cuestionarios individuales aplicados fue el mismo que el número de personas de 60 años o más identificados como residentes habituales del hogar. En el operativo de campo se visitaron 2304 viviendas para lograr 1998 entrevistas completas. En ésas se lograron 2434 entrevistas individuales completas.

\section{Análisis de componentes principales}

El ACP es una técnica estadística de síntesis de la información o reducción de la dimensión (número de variables). Es decir, ante un banco de datos con muchas variables, el objetivo será reducirlas a un menor número perdiendo la menor cantidad de información posible. Es decir, dadas $n$ observaciones de $p$ variables, analizar si es posible representar adecuadamente esta información con un número menor de variables construidas como combinaciones lineales de las originales. Considera una serie de variables $\mathrm{X}_{1}, \mathrm{X}_{2}, \ldots, \mathrm{X}_{\mathrm{p}}$ sobre un grupo de objetos o individuos y se trata de calcular, a partir de ellas, un nuevo conjunto $\mathrm{Y}_{1}, \mathrm{Y}_{2}, \ldots, \mathrm{Y}_{\mathrm{p}}$ no correlacionadas entre sí, cuyas varianzas vayan decreciendo progresivamente.

Cada $y_{j}$ para toda $j=1, \ldots, n$ es una combinación lineal de $\mathrm{X}_{1}, \mathrm{X}_{2}, \ldots$, $\mathrm{X}_{\mathrm{p}}$ originales, es decir: 
$y_{j}=a_{\mathrm{j} 1} X_{1}+a_{\mathrm{j} 2} X_{2}+\ldots+a_{\mathrm{jn}} X_{\mathrm{p}}=\mathrm{a}_{\mathrm{j}} \mathrm{X}$

Donde $a_{j}=a_{j 1}, a_{j 2}, \ldots, a_{j n}$ es un vector de constantes.

El primer componente se calcula eligiendo $a_{1}$ de modo que $y_{1}$ tenga la mayor varianza posible, sujeta a la restricción:

$$
\sum_{k=1}^{p} a_{k 2}^{2}=1
$$

El segundo, se calcula obteniendo $a_{2}$ de modo que la variable obtenida, $y_{2}$ no este correlacionada con $y_{1}$, y así sucesivamente, de manera que las variables obtenidas vayan teniendo cada vez menor varianza (De la Fuente, 2011).

Como se ha mencionado, la calidad de vida en general depende tanto de factores objetivos como subjetivos, y en esta investigación el índice de calidad de vida del adulto mayor se construyó considerando cuatro dimensiones: a) condiciones de la vivienda, b) ingreso, c) bienestar subjetivo y d) condiciones de salud; y se utilizaron ocho variables: i) derecho a recibir el pago de una pensión, ii) frecuencia con la que el adulto mayor reporta disfrutar de la vida, iii) nivel de felicidad reportado, iv) percepción de la salud, v) posesión de bienes duraderos, vi) derechohabiencia, vii) material de paredes y viii) material del techo en la vivienda.

El ACP arrojó un primer componente que contiene cerca de 33 por ciento de la varianza total, por lo que ésta pasa a ser el índice de calidad de vida buscado para los adultos mayores, ya que explica la variabilidad del conjunto de las ocho variables mencionadas. La viabilidad del ACP se verificó a través de dos estadísticos: Test de esfericidad de Barlett, que permite contrastar la hipótesis de que la matriz de correlaciones es una de identidad y el Test de Kaiser-Meyer-Olkin (KMO), que mide la idoneidad de los datos para realizar un análisis factorial, comparando los valores de los coeficientes de correlación observados con los de correlación parcial. El estadístico KMO varía entre cero y uno, los valores menores a 0.5 indican que no debe utilizarse el ACP con los datos que se estén empleando.

Una vez construido dicho índice, se aplicó el método de estratificación de Dalenius y Hodges, que de acuerdo con García (2013) es el método más utilizado en el análisis de información de las condiciones relativas de vida (llámese índice de marginación, vulnerabilidad social, niveles de bienestar que son índices multidimensionales que incluyen valores positivos y negativos), y que permite obtener una agrupación lo más homogénea posible entre las observaciones de una base de datos, así como construir tantos 
estratos como uno lo desee, tomando en cuenta la propia distribución de los datos y minimizando la varianza.

\section{Resultados}

En la Tabla 3 se muestran las variables empleadas para el ACP, así como los porcentajes respectivos, donde se observa que 82.38 por ciento de los adultos mayores carecen del goce de una pensión o jubilación, 95.81 por ciento de los adultos mayores mencionó sentirse feliz algunas veces o siempre; respecto a la percepción de salud, apenas 15.64 por ciento señaló un estado excelente, puesto que la mayoría reportó estar bien o regular y apenas el nueve por ciento lo calificó como malo.

En cuanto a sus viviendas, 72.76 por ciento de adultos mayores cuenta con espacios apropiados (paredes de materiales durables) y 62.53 por ciento reportó condiciones duraderas del techo de su casa; que por otra parte, 42.34 por ciento carecen de bienes duraderos. Por último, se destaca que apenas 44.19 por ciento tiene acceso a una institución de salud que no sea el Seguro Popular para recibir atención médica (ESEDEM 2008).

En México se mantiene un sistema segmentado y fragmentado en el cual el seguro social laboral es obligatorio y público, mientras que el Sistema Nacional de Protección Social en Salud (SNPSS), mejor conocido como el Seguro Popular (SP), es voluntario y sólo la población sin seguro social es susceptible a ser afiliada. El SP se financia con contribuciones fiscales de los gobiernos federal y estatales, además ofrece un paquete de servicios - el Catálogo Universal de Servicios de Salud (CAUSES) - muy inferior a los seguros médicos de los institutos, y sólo incluye un reducido número de enfermedades de alto costo (Laurell, 2013). Por tal motivo, y dadas las carencias en infraestructura y personal del SP, ser afiliado al SP no es sinónimo de derechohabiencia.

Empleando la estratificación mencionada se formaron cinco grupos para la calidad de vida: muy baja, baja, regular, alta y muy alta. Se analizaron en total 1949 casos y los resultados de dicho análisis permiten afirmar que 42.6 por ciento de la población presenta calidad de vida de alta a muy alta, cifra que pareciera muy alentadora; sin embargo, 25 por ciento del total de los adultos mayores en estudio caen en la categoría de nivel medio, y el resto, 32.4 por ciento, corresponde a niveles bajo y muy bajo como se observa en la Figura 4. Dada esta situación, resulta interesante conocer el comportamiento de las variables sociodemográficas, económicas y de la salud, a fin de proponer o mejorar políticas públicas encaminadas a incrementar el bienestar de los adultos mayores. 
Tabla 3: Variables para la construcción del Índice de calidad de vida

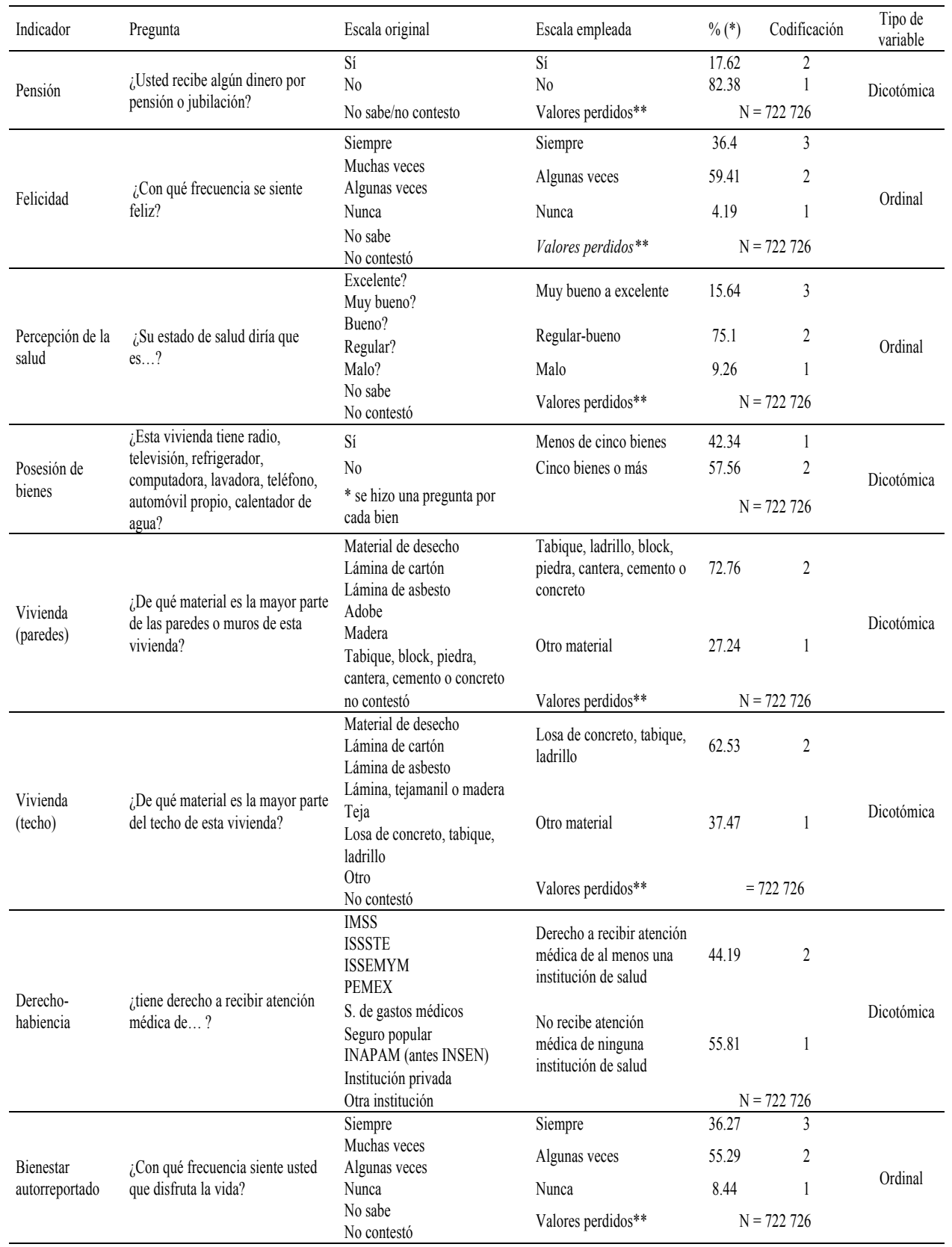

* porcentajes con factores de expansión.

** estos valores no fueron considerados en esta investigación, por lo que se omitieron dichas observaciones.

Fuente: elaboración propia a partir de datos provenientes de la ESEDEM (2008). 
Figura 4: Distribución porcentual del nivel de calidad de vida en los adultos mayores del Estado de México

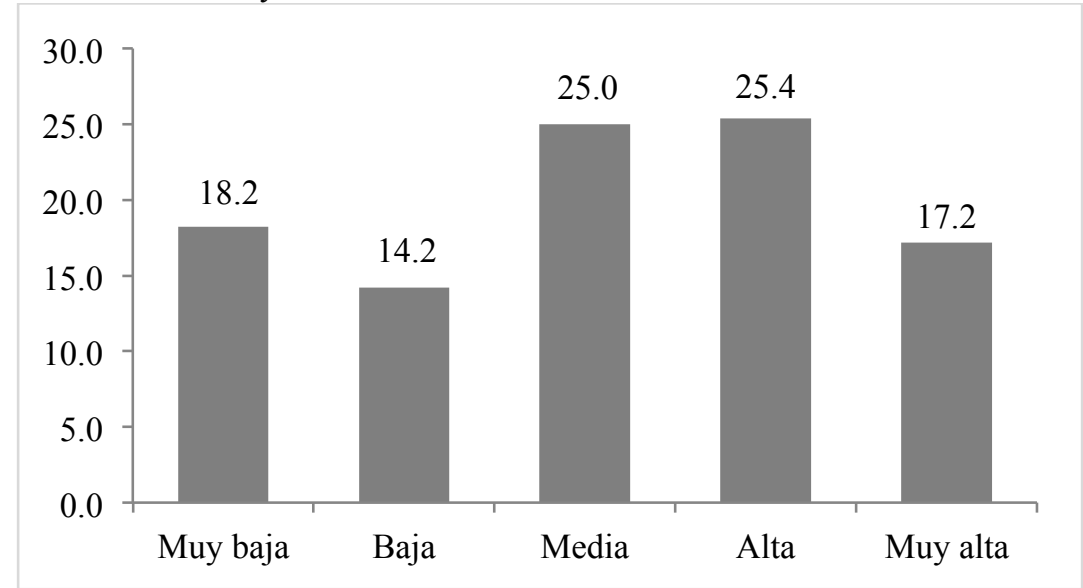

Fuente: elaboración propia con base en ESEDEM, 2008.

A continuación se analiza la frecuencia en los niveles de bienestar, por cada variable utilizada para su construcción se señala que los adultos mayores con derecho al pago de una pensión, con buenas condiciones de vivienda (techo y paredes de materiales adecuados y posesión de bienes duraderos), con derecho a recibir atención médica por alguna institución pública o privada (no Seguro Popular), que disfrutan de la vida con frecuencia y con buenos estados de salud y felicidad reportados, corresponden a individuos con una calidad de vida alta y muy alta. En la Tabla 4 los porcentajes que se muestran omiten a los individuos que se clasificaron con calidad de vida media, baja y muy baja y se puede apreciar lo antes comentado. Esto se hace porque se busca identificar las condiciones y características de los adultos mayores calificados con niveles altos de calidad de vida para marcar una directriz en la instrumentación de políticas que busquen atender y mejorar las mismas condiciones en la población con niveles bajos de calidad de vida.

Resulta interesante cuestionarse por qué motivos un amplio porcentaje de los adultos mayores muestra buena calidad de vida, pese a que gran número de ellos también presenta carencias como la falta de servicios médicos, ingresos por concepto de pensión o vivienda digna, pero como se mencionó, en la calidad de vida juegan factores tanto objetivos como subjetivos y variables como el apoyo percibido, redes familiares, recreación o no padecer enfermedades crónicas incapacitantes son elementos aún más relevantes que factores económicos o carencias materiales. 
Tabla 4: Distribución de calidad de vida por variables empleadas para la construcción del índice*

\begin{tabular}{llr}
\hline Variables & Indicador & Porcentaje \\
\hline \multirow{2}{*}{ Derechohabiencia } & Sí & 84.5 \\
& No & 15.5 \\
\hline \multirow{2}{*}{ Posesión de bienes } & Cuatro bienes o más & 92.3 \\
& Menos de cuatro bienes & 7.7 \\
\hline \multirow{3}{*}{ Felicidad } & Siempre & 42.9 \\
& Algunas veces & 54.3 \\
& Nunca & 2.8 \\
\hline \multirow{2}{*}{ Techo } & Adecuado & 95.7 \\
& No adecuado & 4.3 \\
\hline \multirow{2}{*}{ Paredes } & Adecuado & 98.3 \\
& No adecuado & 1.7 \\
\hline \multirow{2}{*}{ Salud } & De regular a excelente & 90.0 \\
& Malo & 10.0 \\
\hline \multirow{2}{*}{ Pensión } & Sí & 39.0 \\
& No & 61.0 \\
\hline \multirow{2}{*}{ Disfruta de la vida } & Siempre & 44.8 \\
& Algunas veces & 50.1 \\
& Nunca & 5.1 \\
\hline
\end{tabular}

* Sólo considera personas que clasificaron en niveles de calidad de vida alto y muy alto.

Fuente: elaboración propia con base en ESEDEM, 2008.

\section{Análisis descriptivo de las variables suplementarias}

La idea es que una vez obtenido el índice de calidad de vida se asocie con distintas variables, llamadas suplementarias o ilustrativas, que son otras medidas de los individuos de la muestra, porque no intervienen en la formación de los componentes (González et al., 2002). Entonces, una vez calculado el índice de calidad de vida para los adultos mayores, es conveniente analizar las características sociodemográficas y de la salud que aborda esta investigación y la relación que guarda cada una de ellas con el índice de calidad de vida. 


\section{Características sociodemográficas}

De los 1949 adultos mayores considerados en el estudio, 51.4 por ciento corresponde a personas de sexo femenino. Para efectos de un mejor análisis para la variable edad se conformaron por grupos, en la Figura 5 se muestra que el rango de edad de los 60 a los 74 años alberga 70 por ciento del total de los adultos mayores, aunque, dadas las tendencias en el envejecimiento poblacional, este rango se irá recorriendo hacia edades más avanzadas.

En lo que respecta al nivel de calidad de vida por sexo, el género masculino presenta mejores condiciones. Se observa, en la Tabla 5, que 45.45 por ciento de los hombres presentan calidad de vida alta y muy alta, mientras que el porcentaje es menor en las mujeres, con 39.88 por ciento; de modo que es posible asumir que la variable sexo tiene cierta influencia en la calidad de vida.

Figura 5: Distribución porcentual por grupos de edad de la población de 60 años o más

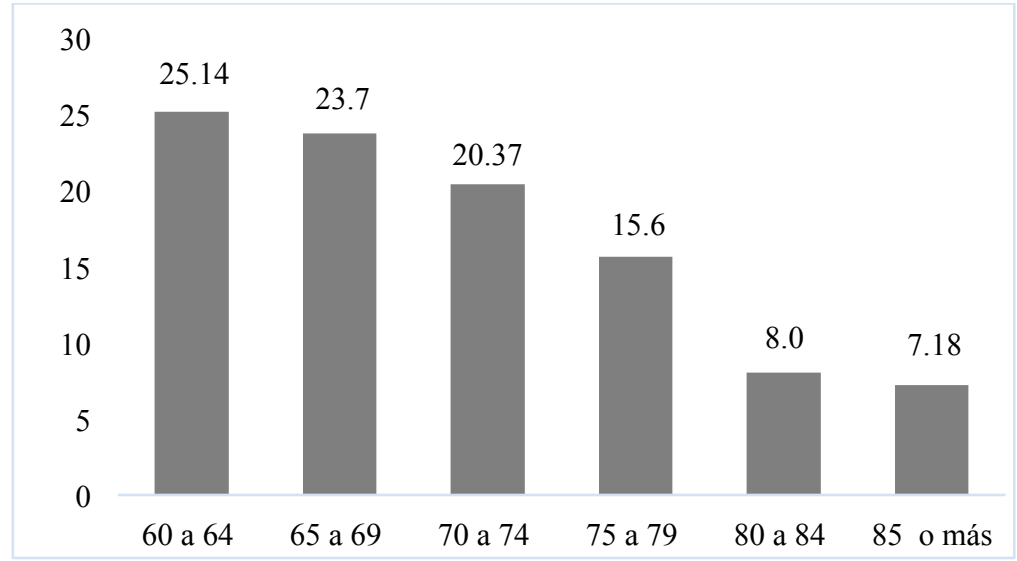

Fuente: elaboración propia con base en ESEDEM, 2008.

Otra variable sociodemográfica por analizar es el estado civil, a partir de los datos de la ESEDEM (2008), del total de adultos mayores en estudio, 59 por ciento reportó vivir en pareja (casados o en unión libre), diez por ciento no vive en pareja (solteros, separados o divorciados) y el resto corresponde a adultos mayores viudos. 
Tabla 5: Distribución porcentual de calidad de vida según el sexo

\begin{tabular}{lrr}
\hline & Hombre & Mujer \\
\hline Nivel de calidad de vida & & \\
Muy baja & 17.02 & 19.34 \\
Baja & 14.06 & 14.36 \\
Media & 23.47 & 26.42 \\
Alta & 23.15 & 27.52 \\
Muy alta & 22.30 & 12.36 \\
Total & 100.00 & 100.00 \\
\hline
\end{tabular}

Fuente: elaboración propia con base en ESEDEM, 2008.

En la Figura 6 se observa que la mayoría de los hombres (78.01 por ciento) viven en pareja, a diferencia de las mujeres con 42.57 por ciento; por otra parte, hay un mayor número de mujeres viudas que hombres, principalmente a causa de la esperanza de vida en el sexo femenino.

Figura 6: Distribución porcentual del estado civil de la población de 60 años o más según el sexo

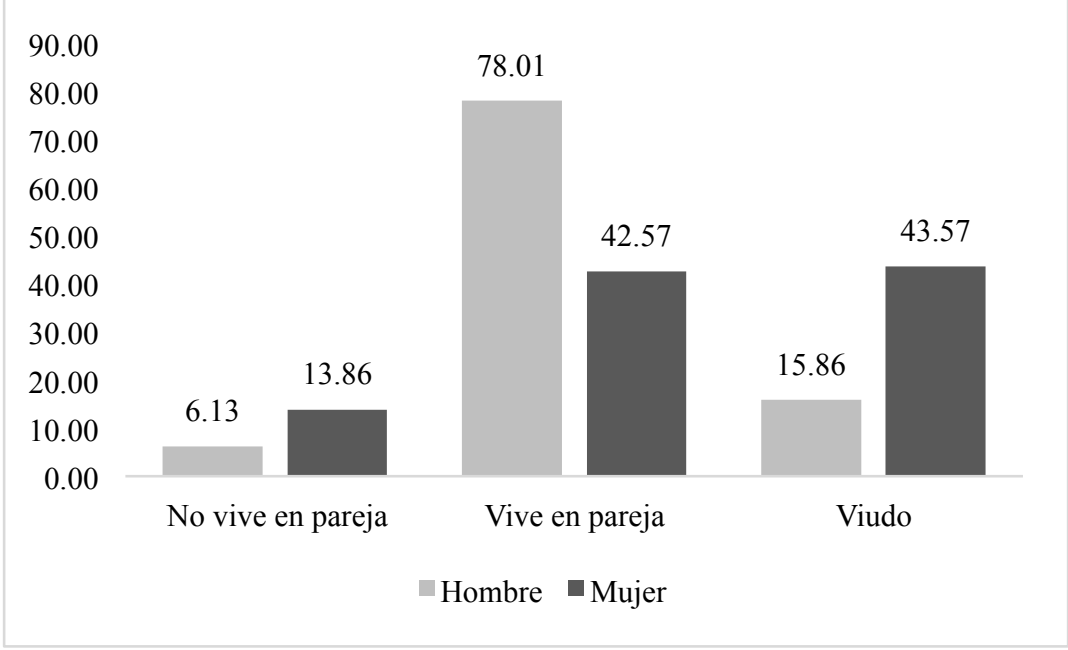

Fuente: elaboración propia con base en ESEDEM, 2008.

Las relaciones sociales de las personas impactan en su salud mental y psicológica, pues, mantener contacto con las personas que le rodean es esencial. De acuerdo con Montoya y Montes de Oca (2010) envejecer en compañía de otras personas genera mayores condiciones de resistencia y 
recuperación, tanto de enfermedades como de crisis emocionales y económicas. De acuerdo con la CEPAL:

Las relaciones familiares son cruciales para la manutención y el bienestar de la población envejecida. Entre éstas destaca el estado civil, en específico la convivencia en pareja. Contar con el cónyuge representa beneficios primordiales como son la satisfacción sentimental y psicológica de la compañía, la posibilidad de atención y cuidados mutuos y la oportunidad de apoyo material y moral. En el otro lado de la balanza, se ha comprobado que la soledad es un gran factor de depresión en la vejez, cuestión que afecta particularmente a los hombres que se quedan solos. Asimismo, la dependencia para los cuidados y el sustento sobre otros miembros de la familia no es tan constante ni tan confiable como la de la propia pareja. De esta manera, una marca psíquica y social del envejecimiento individual es el estado especial de soledad y falta de apoyo que viene con la viudez, en especial para las mujeres (CEPAL, 2002: 24).

En la Tabla 6 se aprecia que un alto porcentaje de adultos mayores que son viudos presentan niveles de calidad de vida baja y muy baja, del total que viven en pareja, 15.28 y 2.83 por ciento de hombres y mujeres, respectivamente, se clasificaron en la categoría de calidad de vida muy alta. De esta forma se puede confirmar que el estado civil y sexo también influyen en la calidad de vida.

En la Tabla 7 se observa que 62.99 por ciento de adultos mayores con calidad de vida muy alta reportó vivir en pareja, con esto se confirma lo que dice Montoya y Montes de Oca (2010), respecto a envejecer en compañía de seres queridos.

En cuanto a la escolaridad de la población en estudio, y como se ve en la Figura 7, 57.31 por ciento tiene primaria o secundaria; 37.51 por ciento de los adultos mayores no cuenta con algún grado de estudios aprobado y apenas 5.18 por ciento de ellos tiene alguna carrera técnica, profesión o posgrado.

En la Tabla 8 es evidente la relación que existe entre el ámbito de bienestar y el educativo; se aprecia que a mayor educación, mejor calidad de vida y los porcentajes se inclinan hacia el sexo masculino. Por ejemplo, 65.38 por ciento de los hombres que tienen una profesión se clasificaron en el rango de calidad de vida muy alto, mientras que 43.75 por ciento de las mujeres lo hizo; por otro lado, 27.24 por ciento de los hombres que no tuvieron instrucción académica tienen calidad de vida muy baja y 28.98 por ciento para el caso de las mujeres sin nivel de escolaridad. 


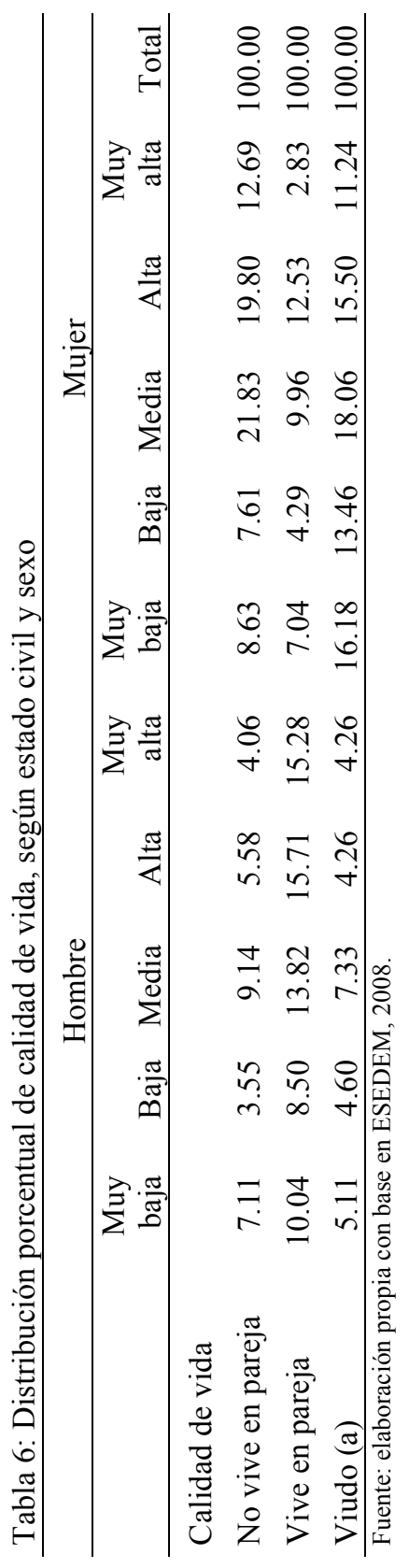


Tabla 7: Distribución de calidad de vida, según su estado civil

\begin{tabular}{lrrrr}
\hline & $\begin{array}{r}\text { No vive } \\
\text { en pareja }\end{array}$ & $\begin{array}{r}\text { Vive } \\
\text { en pareja }\end{array}$ & Viudo (a) & Total \\
\hline Calidad de vida & & & & \\
Muy baja & 8.73 & 56.06 & 35.21 & 100.00 \\
Baja & 7.94 & 53.79 & 38.27 & 100.00 \\
Media & 12.53 & 56.88 & 30.60 & 100.00 \\
Alta & 10.10 & 66.46 & 23.43 & 100.00 \\
Muy alta & 9.85 & 62.99 & 27.16 & 100.00 \\
\hline
\end{tabular}

Fuente: elaboración propia con base en ESEDEM, 2008.

Tabla 8: Distribución porcentual de calidad de vida, según nivel de escolaridad y sexo

\begin{tabular}{lrrrrrrrr}
\hline & \multicolumn{2}{c}{ Ninguno } & \multicolumn{3}{c}{ Educación básica } & \multicolumn{2}{c}{ Bachillerato } & \multicolumn{2}{c}{ Profesión } \\
\hline & Hombre & Mujer & Hombre & Mujer & Hombre & Mujer & Hombre & Mujer \\
\hline Muy baja & 27.24 & 28.98 & 13.73 & 12.65 & 0.00 & 0.00 & 0.00 & 0.00 \\
Baja & 19.71 & 18.58 & 12.44 & 11.45 & 4.55 & 2.70 & 0.00 & 12.50 \\
Media & 24.01 & 26.77 & 24.56 & 27.31 & 0.00 & 10.81 & 11.54 & 25.00 \\
Alta & 20.79 & 20.13 & 23.91 & 33.94 & 31.82 & 35.14 & 23.08 & 18.75 \\
Muy alta & 8.24 & 5.53 & 25.36 & 14.66 & 63.64 & 51.35 & 65.38 & 43.75 \\
Total & 100.00 & 100.00 & 100.00 & 100.00 & 100.00 & 100.00 & 100.00 & 100.00 \\
\hline Fuente: elaboración propia con base en ESEDEM, 2008. & & & & &
\end{tabular}

Figura 7: Distribución porcentual de la escolaridad de la población de 60 años o más

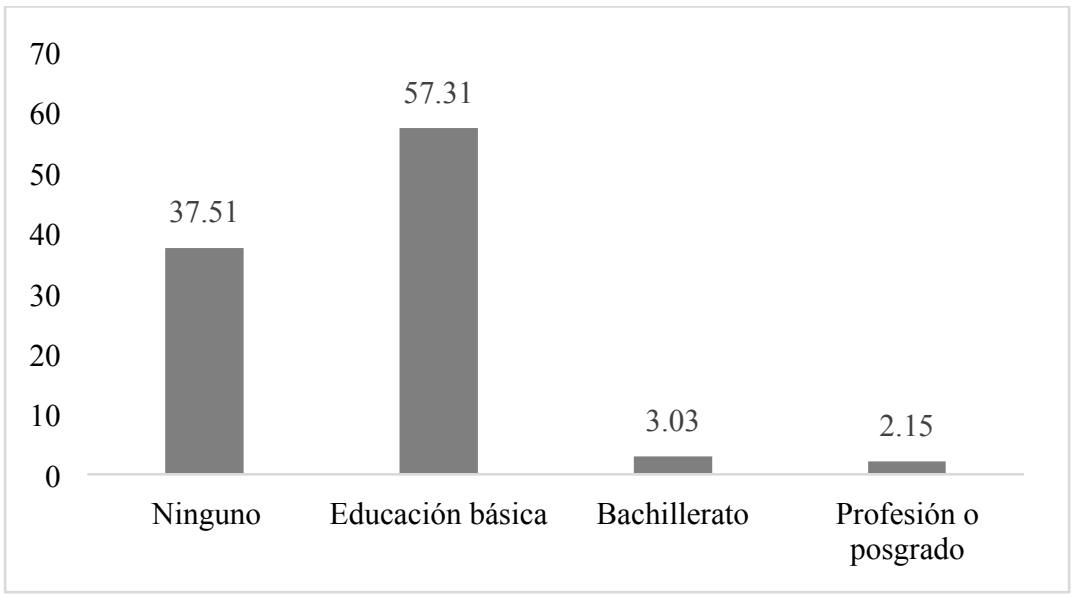

Fuente: elaboración propia con base en ESEDEM, 2008. 
Asimismo, Rodríguez (2007) afirma que uno de los factores de mayor significancia en la calidad de vida del adulto de 60 años o más es la escolaridad y sostiene que su grado de satisfacción es mejor con el apoyo social percibido y con el uso del tiempo libre. En otra investigación se menciona que los ancianos con menor escolaridad e ingreso presentan más dificultades de acceso a oportunidades de ocio y diversión, lo que influye en sus condiciones de salud y, por ende, en su bienestar (Mara dos Santos et al., 2013).

\section{Apoyo percibido}

Existen casos en los que se ve al adulto mayor como una carga económica para la familia y la sociedad, pues se convierte en un individuo que consume sin producir. Generalmente el jubilado disminuye sus ingresos y, al no trabajar, una serie de implicaciones se desatan y su capacidad física y mental se deteriora (Cuenca, 2008).

Por otra parte, Murillo y Venegas (2011) afirman que una proporción significativa de adultos mayores es económicamente activa y para numerosos hogares mexicanos es el único ingreso familiar derivado del trabajo. En la Tabla 9 se observa que conforme la edad aumenta el apoyo percibido también se incrementa, 20.41 por ciento de los adultos de entre 60 y 64 años sí reciben algún tipo de apoyo, mientras que 57.86 por ciento mayores de 85 años reciben alguna ayuda, esto debido a que las personas de mayor edad son quienes se encuentran más vulnerables y necesitan mayores cuidados y apoyo de terceras personas.

Tabla 9: Distribución porcentual de apoyo percibido por parte del gobierno, según por grupos de edad*

\begin{tabular}{lccc}
\hline & No & Sí & Total \\
\hline Grupo de edad & & & \\
60 a 64 años & 79.59 & 20.41 & 100.00 \\
65 a 69 años & 75.76 & 24.24 & 100.00 \\
70 a 74 años & 54.91 & 45.09 & 100.00 \\
75 a 79 años & 46.05 & 53.95 & 100.00 \\
80 a 84 años & 46.79 & 53.21 & 100.00 \\
85 años o más & 42.14 & 57.86 & 100.00 \\
\hline * Apoyo de tipo económico o asistencial. & & \\
Fuente: elaboración propia con base en ESEDEM, 2008. & &
\end{tabular}


En la Tabla 10 se observa que en las categorías de calidad de vida de muy baja a regular se presenta un mayor número de adultos mayores que reciben algún tipo de ayuda (apoyo gubernamental), mientras que en las de bienestar alto y muy alto sucede lo contrario. De los adultos mayores que reciben algún apoyo por parte del gobierno, 65.92 por ciento tienen una calidad de vida muy baja; en contraste, 10.15 por ciento tienen calidad de vida muy alta. La población adulta mayor dependiente de los apoyos, y con mala situación de bienes, muestra serias desventajas, como baja escolaridad, viudez o que habitan en zonas rurales, por lo que estas desventajas tienen un efecto directo sobre su seguridad económica, generando dependencia y afectando su calidad de vida (Madrigal, 2010).

Tabla 10: Distribución porcentual de calidad de vida, según si recibe o no apoyo del gobierno

\begin{tabular}{lrrr}
\hline & No recibe apoyo & Sí recibe apoyo & Total \\
\hline Calidad de vida & & & \\
Muy baja & 34.08 & 65.92 & 100.00 \\
Baja & 46.93 & 53.07 & 100.00 \\
Media & 63.45 & 36.55 & 100.00 \\
Alta & 74.55 & 25.45 & 100.00 \\
Muy alta & 89.85 & 10.15 & 100.00 \\
\hline
\end{tabular}

Fuente: elaboración propia con base en ESEDEM, 2008.

\section{Características de la salud}

"La salud es un estado de completo bienestar físico, mental y social, y no solamente la ausencia de afecciones o enfermedade" (Preámbulo de la Constitución de la Organización Mundial de la Salud). En cuanto al derecho a recibir atención médica por alguna institución de salud, apenas 44.19 por ciento (Tabla 3) de la población en estudio tiene cobertura de servicios médicos, siendo también un mayor porcentaje de hombres con este derecho.

Con la información de la Tabla 11 es posible hacer una comparación entre dos categorías del nivel de bienestar, prácticamente el total de adultos mayores cuya calidad de vida es muy baja no tiene acceso a la atención médica por alguna institución, mientras que en la categoría de calidad de vida muy alta casi 100 por ciento cuenta con servicios médicos por lo que se puede decir que esta es una variable que también tiene alto impacto en su calidad de vida. 
Por otra parte, 58 por ciento de los adultos mayores padecen algún Problema para llevar a cabo Actividades de la Vida Diaria (PAVD), de los cuales el mayor porcentaje son mujeres, debido a la esperanza de vida que tienen y a las desventajas con que llegan a la vejez, a diferencia de los hom-

Tabla 11: Distribución porcentual de calidad de vida según condición de derechohabiencia

\begin{tabular}{lrrr}
\hline & Derechohabiente & No derechohabiente & Total \\
\hline Calidad de vida & & & \\
Muy baja & 0.85 & 99.15 & 100.00 \\
Baja & 12.27 & 87.73 & 100.00 \\
Media & 21.56 & 78.44 & 100.00 \\
Alta & 74.34 & 25.66 & 100.00 \\
Muy alta & 99.40 & 0.60 & 100.00 \\
\hline
\end{tabular}

Fuente: elaboración propia con base en ESEDEM, 2008.

bres (con base en ESEDEM, 2008). En la Tabla 12 se observa que 63.38 por ciento de adultos mayores con al menos un PAVD tiene calidad de vida muy baja, en comparación con 53.43 por ciento de los adultos mayores que tienen muy alta calidad de vida.

Una cifra que resulta preocupante es de los que padece al menos una enfermedad crónico degenerativa, pues casi 64 por ciento de la población presenta esta característica, con las particularidades de que la mayoría son mujeres y que la presencia de éstas no excluye a ningún grupo de edad, como se aprecia en la Tabla 13.

Tabla 12: Distribución porcentual de calidad de vida según PAVD

\begin{tabular}{lccc}
\hline & Algún problema & Sin problemas & Total \\
\hline Calidad de vida & & & \\
Muy baja & 63.38 & 36.62 & 100.00 \\
Baja & 56.32 & 43.68 & 100.00 \\
Media & 61.19 & 38.81 & 100.00 \\
Alta & 55.15 & 44.85 & 100.00 \\
Muy alta & 53.43 & 46.57 & 100.00 \\
\hline
\end{tabular}

Fuente: elaboración propia con base en ESEDEM, 2008.

La relación que en un principio se pudiera plantear entre el índice de calidad de vida y el padecimiento de enfermedades crónicas sería que la presencia de alguna enfermedad influiría de manera negativa en su vida; 
sin embargo, del total con una calidad de vida muy alta, 71.34 por ciento padece alguna enfermedad y 50.7 por ciento con al menos una enfermedad crónica tiene calidad de vida muy baja (Tabla 13).

Tabla 13: Distribución porcentual de calidad de vida según condición de padecimiento de enfermedades crónicas

\begin{tabular}{lrrr}
\hline & $\begin{array}{r}\text { Con enfermedades } \\
\text { crónicas }\end{array}$ & $\begin{array}{r}\text { Sin enfermedades } \\
\text { crónicas }\end{array}$ & Total \\
\hline Calidad de vida & & & \\
Muy baja & 50.70 & 49.30 & 100.00 \\
Baja & 57.40 & 42.60 & 100.00 \\
Media & 65.71 & 34.29 & 100.00 \\
Alta & 69.09 & 30.91 & 100.00 \\
Muy alta & 71.34 & 28.66 & 100.00 \\
\hline
\end{tabular}

Fuente: elaboración propia con base en ESEDEM, 2008.

Empero, otras investigaciones concluyen que los ancianos con enfermedades crónicas se sienten más preocupados debido a las dificultades de acceso a otros recursos de salud y el agravamiento de los problemas de salud, lo que repercute sobre su calidad de vida debido a que disminuyen también sus capacidades funcionales (Mara dos Santos, 2013). Asimismo, Ballesteros (1999) plantea que un decremento notable en la salud física tiene inmediatas repercusiones en el funcionamiento psicológico, por lo que estos conceptos constituyen una unidad inseparable.

La discapacidad física en el adulto mayor imposibilita la realización de tareas como un trabajo y tal vez no cuente con un ingreso económico para satisfacer sus necesidades, lo que se traduce en pasar a ser un individuo dependiente de terceras personas. Autores como Kikuchi (2010) menciona que con las condiciones actuales y dado el incremento en la esperanza de vida es necesario reforzar la salud preventiva y modificar el acuerdo social con jubilados para aumentar la etapa de productividad.

\section{Conclusiones}

Esta investigación dio a conocer las características sociodemográficas, económicas y de la salud de las personas adultas mayores del Estado de México. En primera instancia, el cálculo del índice de calidad de vida da una idea general de la situación que vive este grupo etario y dadas las variables empleadas en el análisis de componentes principales resulta que buenas condiciones de vivienda se relacionan de manera importante con 
niveles de calidad de vida altos, sucede lo mismo con los niveles de felicidad reportados y el derecho a recibir atención médica por instituciones de salud. Por el contrario, no tener derechohabiencia y viviendas en malas condiciones, se asocian a niveles bajos de calidad de vida. Lo anterior revela los aspectos en los que se debe prestar mayor atención de este sector de la población.

Analizar la relación que existe entre el índice de calidad de vida y las variables suplementarias hace posible establecer una alta relación entre la calidad de vida, el estado civil, la educación y la condición de derechohabiencia. También se observa que los adultos mayores viudos presentan desventajas en comparación con aquellos que viven en pareja, por lo que es posible confirmar que, en efecto, envejecer en compañía de otras personas puede ayudar a propiciar mejores condiciones de vida y que, además, esto puede ocurrir en mayor medida si las personas comparten lazos afectivos como lo mencionan Montoya y Montes de Oca (2010), por lo que será de suma importancia fomentar una cultura de respeto y apoyo hacia el adulto mayor.

En cuanto a la escolaridad, se afirma que una persona con estudios tiene mayores oportunidades de insertarse en el mercado laboral formal y mejor remunerado, por lo que el alcance de satisfacer sus necesidades es superior al de aquellas personas que no tienen instrucción académica; además de recibir una pensión. En general, cerca de la mitad de la población que se analizó carece de estudios y de ahí que el porcentaje similar se haya clasificado en niveles de bienestar bajos.

Hay diferencias entre las oportunidades que tiene un adulto mayor hombre a una mujer. En general tiene mayores ventajas educativas, posibilidad de un trabajo formal y, con ello, el derecho a acceder a una pensión y contar con servicio médico. La variable edad se relaciona con la de bienestar, pues a medida que una persona envejece sus capacidades físicas también reducen.

Además, existe un número considerable de adultos mayores que presentan al menos una enfermedad crónica, lo que se vuelve alarmante, porque implicarán una creciente demanda de servicios de atención médica, que, a su vez, provocan gastos y desestabilizarían aún más la economía de las familias y del país. En este punto cabe resaltar que el Seguro Popular es limitado, porque hasta ahora sólo atiende algunos padecimientos.

También se aprecia que los apoyos sociales que pudieran recibir por parte del gobierno son bajos, lo que pone en entredicho su finalidad; si bien, carecer de ellos involucraría a un mayor número de adultos mayores 
con niveles de vida desfavorables, distan mucho de incrementar su calidad de vida.

En el Estado de México actualmente se desarrollan programas de atención a este grupo vulnerable, tales como la entrega de canastas alimentarias a personas de 60 a 69 años, así como para mayores de 70; asimismo se impulsa la integración de esta población a la vida productiva a través de cursos de capacitación a fin de mejorar su economía. Existen también apoyos económicos federales como el Programa 70 y más, que consiste en la entrega de 500 pesos mensuales y que se paga cada dos meses; sin embargo, el objetivo de estos programas dista mucho de ofrecer un incremento sustancial en la calidad de vida de este sector.

Cabe señalar que la Ley del Adulto Mayor del Estado de México en su artículo primero refiere lo siguiente: "La presente Ley es de orden público, de interés social y de observancia general en el Estado de México y tiene por objeto garantizar el ejercicio de los derechos de los adultos mayores, así como establecer las bases y disposiciones para su cumplimiento, a efecto de mejorar su calidad de vida" (Ley del Adulto Mayor del Estado de México, 2008), sin embargo, bajo estas circunstancias, los apoyos gubernamentales resultan insuficientes para garantizar un nivel de vida adecuado $\mathrm{y}$, dadas las proyecciones demográficas en materia de envejecimiento, es válido suponer que este tipo de programas no están garantizados, por lo que la pobreza en esta etapa de la vida se agrava.

Ante tal situación, y para solventar sus necesidades de subsistencia, un alto número de personas mayores se mantiene inmerso en el mercado laboral, pero al reducirse sus capacidades físicas pasan a un estado de dependencia y su cuidado recae en las redes familiares, mismas que actualmente son de menor tamaño debido al descenso en la tasa de fecundidad; entonces, las familias tendrán que responsabilizarse no sólo del cuidado de los hijos, sino también de los mayores, y que éstas sean de menor tamaño, implica que haya menos hermanos con quienes compartir el cuidado y la responsabilidad. Aunado a esto, fenómenos migratorios y situaciones de pobreza ponen en duda su capacidad para cargar con la responsabilidad de la protección de este grupo de personas.

Con la información y el análisis de los datos se refleja una seria preocupación en materia de salud, económica, educativa y política, por lo que se resalta la necesidad de abordar el envejecimiento demográfico con planeación y con miras hacia la correcta atención de demandas y necesidades que ya exige este grupo poblacional. Es decir, se debe dar mayor importancia en la creación de políticas enfocadas en la prevención de la salud 
y el bienestar para la población aun en edades no avanzadas, reforzando la estructura social y económica a largo plazo, pues no se debe olvidar que las condiciones asociadas al envejecimiento que requieren atención se multiplican con el paso del tiempo y van acompañadas de una creciente condición de dependencia.

Bajo esta situación es urgente avanzar en materia de seguridad social, así como impulsar la investigación en las áreas relacionadas con el envejecimiento y garantizar, alos aún jóvenes, un trabajo digno que propicie la acumulación suficiente de recursos económicos y con ello un nivel adecuado de vida cuando se llegue a la edad de retiro laboral.

\section{REFERENCIAS BIBLIOGRÁFICAS}

Ballesteros-Fernández, R., 1999, Introducción a la evaluación psicológica II, Pirámide. Madrid.

Busso, Gustavo, 2005, "Pobreza, exclusión y vulnerabilidad social. Usos, limitaciones y potencialidades para el diseño de políticas de desarrollo y de población", en VIII Jornadas Argentinas de Estudios de Población (AEPA), Tandil, Provincia de Buenos Aires.

CEPAL, 2002, Calidad de vida y redes de apoyo social de las personas en edades avanzadas en la ciudad de México. Reunion de expertos en redes de apoyo social a personas adultas mayores: El rol del Estado, la familia y la comunidad. Santiago de Chile. Disponible en http://www.cepal.org/cgi-bin/getProd.asp?xml=/ celade/noticias/paginas/7/11497/P11497.xml\&xsl=/celade/tpl/p18f.xsl\&base=/ tpl/imprimir.xslt

CIEAP, 2008, Encuesta sobre Envejecimiento Demográfico en el Estado de México (ESEDEM 2008), Centro de Investigación y Estudios Avanzados de la Población. Universidad Autónoma del Estado de México. Toluca.

CNPD, 2010, ENADIS, Consejo Nacional para Prevenir la Discriminación. Disponible en http://www.conapred.org.mx/index.php?contenido=pagina\&id=424\&id_ opcion $=436 \&$ op $=436$

CONAPO, 2016, Estimaciones y proyecciones de la población por entidad federativa, México: Consejo Nacional de Población. Disponble en http://www.conapo. gob.mx/es/CONAPO/Proyecciones_Datos, fecha de consulta 29/02/2016.

Cuenca, Ángel, 2008, "Calidad de vida en la tercera edad", en Cuadernos de Bioética, XIX(2), pp. 271-291.

De la Fuente, Santiago, 2011, Componentes principales, Fac. Ciencias Económicas y Empresariales UAM. Disponible en http://www.fuenterrebollo.com/Economicas/ECONOMETRIA/MULTIVARIANTE/ACP/ACP.pdf

García, Iván, 2013, "Modelo para el análisis multidimensional de la pobreza" en Geografia y Sistemas de Información Geográfica, pp. 243-268. 
García, Julio César, 2008, Análisis del bienestar de los adultos mayores en México, Tesis de Maestría, FLACSO, Sede Académica de México. México.

González, Martín et al., 2002, "Una aplicación de análisis de componentes principales en el área educativa”, en Revista Economía, Mérida.

Guzmán, José Miguel y Huenchuan, Sandra, 2006, “Seguridad económica y pobreza en la vejez: tensiones, expresiones y desafíos para el diseño de políticas", en Notas de Población, vol. 33, núm. 83.

Guzmán, José, 2003, Seguridad económica en la vejez: una aproximación inicial, ponencia preparada para Reunión de Expertos en Seguridad Económica del Adulto Mayor, Panamá.

Ham, Roberto, 1998, "Implicaciones del envejecimiento en la planeación del bienestar", en Papeles de Población, núm. 17, julio-septiembre, UAEM/CIEAP. Toluca.

INEGI, 2005, Los adultos mayores en México: perfil sociodemográfico al inicio del siglo XXI, Aguascalientes, México.

INEGI, 2013, ENESS, Instituto Nacional de Estadística y Geografía. Disponible en http://www.inegi.org.mx/est/contenidos/proyectos/encuestas/hogares/modulos/eness/eness2013/default.aspx

INEGI, 2015, Encuesta Intercensal 2015, Instituto Nacional de Estadística y Geografía. Disponible en http://www.beta.inegi.org.mx/proyectos/enchogares/especiales/intercensal/?init=1

Kikuchi-Yokoyama, M., 2010, "Reflexiones sobre la plática de demografía de la seguridad social", en Actuarios trabajando: Revista Mexicana de Investigación Actuarial Aplicada, año 3, núm. 5, pp. 73-79.

Laurell, Cristina, 2013, Impacto del seguro popular en el sistema de salud mexicano, CLACSO. Buenos Aires.

Ley del Adulto Mayor del Estado de México, 2008, Disponible en http://info4. juridicas.unam.mx/adprojus/leg/16/652/2.htm?s= (consultado el 6/08/2008).

Madrigal, Mariana, 2010, "Ingresos y bienes en la vejez, un acercamiento a la configuración de la seguridad económica de los adultos", en Papeles de Población, núm 63, enero-marzo, UAEM/CIEAP. Toluca.

Mara Dos Santos, Darlene et al., 2013, “Características sociodemográficas y calidad de vida de ancianos con hipertensión arterial sistémica que viven en la zona rural: importancia del papel del enfermero", en Rev. Latino-Am. Enfermagem, 21(12).

Millán, José Carlos, 2011, "Envejecimiento y calidad de vida", en Revista Galega de Economía, vol. 20, pp. 1-13.

Montoya-Arce, Bernardino Jaciel y Montes de Oca, Hugo, 2010, "Los adultos mayores del Estado de México en 2008: un análisis sociodemográfico", en Papeles de Población, núm 65, julio-septiembre, UAEM/CIEAP. Toluca. 
Murillo, Sandra y Venegas, Francisco, 2011, "Cobertura de los sistemas de pensiones y factores asociados al acceso a una pensión de jubilación en México", en Papeles de Población, núm 67, enero-marzo, UAEM/CIEAP, Toluca.

Nava-Bolaños, I. y Ham, Roberto, 2014, "Determinantes de la participación laboral de la población de 60 años o más en México", en Papeles de Población, núm. 81, julio-septiembre, UAEM/CIEAP. Toluca.

Ochoa, Sara María, 2011, “Apuntes para la conceptualización y la medición de la calidad de vida en México", en C. D. Pública, Bienestar y Calidad de vida en México, pp. 15-68, México.

OMS, 2002, "Programa envejecimiento y ciclo vital. Envejecimiento activo: un marco político", en Revista Española de Geriatría y Gerontología, 37(S2), pp. $74-105$.

OMS, 2012, Envejecimiento en el siglo XXI: una celebración y un desafio, Fondo de Población de las Naciones Unidas, (UNFPA), HelpAge International, Londres y Nueva York.

Ordorica, Manuel, 2010, "Las proyecciones de la población hasta la mitad del siglo XXI", en Los grandes problemas de México, El Colegio de México. México, pp. 29-51.

Ordorica, Manuel, 2012, “¿Cómo aprovechar el éxito de la política de población del último cuarto del siglo XX para enfrentar los nuevos retos demográficos del XXI?", en Papeles de Población, vol. 18, núm. 74.

Organización Panamericana de la Salud, 2002, "Promover un estilo de vida para las personas adultas mayores", en Guía Regional para la promoción de la actividad fisica, Washington.

Ovalle, Omar y Martínez, Javier, 2006, "La calidad de vida y la felicidad. Contribuciones a la Economía", en Contribuciones a la economía. Disponible en http:// www.eumed.net/ce/

Puig, Montserrat; Rodríguez, Nuria; Farrás, Jaume y Lluch, María Teresa, 2011, "Calidad de vida, felicidad y satisfacción con la vida en personas ancianas de 75 años atendidas en un programa de atención domiciliaria", en Rev. Latino-Am. Enfermagem, vol. 19(3).

Ramírez, Marta Lucía, 2002, "Reseña de guía sobre salud y población”, en Heredia-Benítez, Rodolfo, Ramírez-Giraldo, Marta Lucía y Vivas-Benítez, Alejandro (editores), en Revista Gerencia y Políticas de Salud, vol. 1, núm. 2.

Robles, Yolanda et al., 2010, "Índice de calidad de vida: Validación en una muestra peruana", en Anales de Salud Mental, XXVI (2), pp. 33-43.

Rodríguez-Fijóo, N., 2007, "Factores que influyen sobre la calidad de vida de los anciones que viven en geriátricos" en Psicología y Psicopedagogía, VI(17).

Romero, José Tomás, 2005, “Discriminación y adultos mayores: un problema mayor”, en El Cotidiano, núm. 134, noviembre-diciembre, 2005, pp. 56-63. 
Tuirán, Rodolfo, 1999, “Desafíos del envejecimiento demográfico en México”, en Consejo Nacional de Población, pp. 15-22, México.

Vera, Martha, 2007, "Significado de la calidad de vida del adulto mayor", en Anales de la Facultad de Medicina, 68(3), pp. 284-290.

Vinaccia, Stefano y Orozco, Lina María, 2005, “Aspectos psicosociales asociados con la calidad de vida de personas con enfermedades crónicas Diversitas", en Perspectivas en Psicología, vol. 1, núm. 2, julio-diciembre, Universidad Santo Tomás Bogotá. pp. 125-137, Colombia.

Welti, Carlos, 2013, "Política social y envejecimiento", en Papeles de Población, núm. 77, julio-septiembre, UAEM/CIEAP. Toluca.

World Health Organization, 1948, Official Records of the World Health Organization, núm. 2, New York.

Zetina, María Guadalupe, 1999, “Conceptualización del proceso de envejecimiento”, en Papeles de Población, núm. 19, enero-marzo, UAEM/CIEAP. Toluca.

\section{INFORMACIÓN CURRICULAR DE LOS AUTORES}

\section{Zuriel Soria Romero}

Egresado de la Licenciatura en Actuaría de la Facultad de Economía, Universidad Autónoma del Estado de México. Ha participado como becario en diversos proyectos de investigación relacionados con la población adulta mayor en el Estado de México. Sus intereses son los temas de envejecimiento y dependencia, desempleo y seguridad social.

Dirección electrónica: zuriel_soria@hotmail.com

\section{Bernardino Jaciel Montoya Arce}

Es Licenciado, Maestro y Doctor en Sociología por la Universidad Nacional Autónoma de México (UNAM). Pertenece al Sistema Nacional de Investigadores Nivel I, cuenta con Perfil Deseable PROMEP. Actualmente es Coordinador del Centro de Investigación y Estudios Avanzados de la Población de la Universidad Autónoma del Estado de México (CIEAPUAEM). Entre sus publicaciones recientes se encuentran Demografía indígena en el Estado de México (coautor), 2013; Análisis demográfico del envejecimiento en el Estado de México (compilador), UAEM, 2011 y "La educación indígena en el Estado de México", en Papeles de Población, 2013.

Dirección electrónica: bjmontoyaa@uaemex.mx

Artículo recibido el 1 de junio de 2016 y aprobado el 25 de julio de 2016. 